\title{
Increased detection of enterovirus A71 infections, Germany, 2019
}

Sindy Böttcher ${ }^{1}$, Sabine Diedrich ${ }^{1}$, Kathrin Keeren², the Laboratory Network for Enterovirus Diagnostic (LaNED) ${ }^{3}$

1. National Reference Centre for Poliomyelitis and Enteroviruses, Robert Koch Institute, Berlin, Germany

2. Secretary of the National Commission for Polio Eradication in Germany, Robert Koch Institute, Berlin, Germany

3. The members of the network are listed at the end of the article

Correspondence: Sindy Böttcher (BoettcherS@rki.de)

We report on the increased circulation of enterovirus A71 in Germany in 2019. Strains were mainly identified in hospitalised patients with suspected aseptic meningitis/encephalitis. Molecular analysis showed co-circulation of EV-A71 sub-genogroups $\mathrm{C}_{1}$ and $\mathrm{C}_{4}$, a signal for physicians and public health authorities to include/intensify EV diagnostic in patients showing signs of aseptic meningitis, encephalitis or acute flaccid paralysis/myelitis.

In Europe, enterovirus A71 (EV-A71) has mainly been detected sporadically in patients with neurological disorders. C2 has been the predominant sub-genogroup during the last decade [1]. A multi-recombinant $C_{1}$ lineage ( $C_{1}$-like) emerged in 2015 in Germany [2-4]. Since then, it was detected in sporadic cases with severe neurological disease in several European countries [4-6], as well as in an outbreak in Catalonia, Spain in 2016 [7,8]. In south-eastern Asia, EV-A71, sub-genogroup $\mathrm{C}_{4}$ strains have been mainly associated with severe and even fatal courses of hand, foot and mouth disease (HFMD) in children [1].

Here we report on an increase of EV-A71 C1-like strains in Germany in 2019 and first detections of EV-A71 C4 strains in patients with neurological disease and HFMD.

\section{Enterovirus surveillance in Germany}

In Germany, enterovirus surveillance (EVSurv) is conducted within the framework of the Global Polio Eradication Initiative and provides continuous data on circulating enteroviruses since 2006. Stool or cerebrospinal fluid samples of hospitalised patients with signs of aseptic meningitis/encephalitis and/or acute flaccid paralysis are analysed within a quality-controlled laboratory network (LaNED). Laboratory results and pseudonymised patient data are reported to the national public health institute (Robert Koch Institute; RKI). Data are analysed and the results are publically available at evsurv.rki.de. Previous data on EV-A71 circulation suggested that EV-A71 strains circulate in upsurges every 3 years, with 2010 and 2013 being epidemic years in Germany [2].

Typing of enterovirus (EV) strains is performed according to the individual network laboratory algorithm, by sequencing the complete or partial $\mathrm{VP}_{1}$ or $\mathrm{VP}_{4} / \mathrm{VP}_{2}$ region and/or virus isolation and typing by neutralisation assay. Assignment of the EV-A71 strains to one of the seven known sub-genogroups was performed using the Enterovirus Genotyping Tool [9] and/or neighbourjoining algorithm-based phylogenetic tree analysis. All sequences were submitted to GenBank under accession numbers (MN397830-MN397906).

Since 2006 , ca 2,500 samples have been tested annually within the EVSurv for a total of ca 34,000 analysed since that time. Of these, $25-30 \%$ were EV-positive. Overall, echovirus 30 was the most prevalent EV type $(n=2,194)$, followed by echovirus $6(n=775)$ and EV-A71 $(n=496)$. A total of 32 EV-A71 positive samples were documented in 2017 and 40 in 2018.

\section{Enterovirus A71 detections through enterovirus surveillance}

From January 2019 to the end July 2019, 38 EV-A71 positive patients were registered, including one fatal case. EV-A71-positive patients were observed in March $(n=2)$, April $(n=3)$, May $(n=6)$, June $(n=14)$ and July $(n=13)$. Besides EV-A71, CV-B5 was the second most common EV type $(n=24)$ within the EVSurv this year. Typing results are still pending for $35 \%(73 / 209)$ of EV-positive samples.

Of the 38 EV-A71 strains detected, 26 were analysed further at the National Reference Centre for Poliomyelitis and Enteroviruses (NRZ PE). Sequencing of the VP1 region was done as described previously [2]. Twentyone strains were assigned to $\mathrm{C}_{1}$-like, four to sub-genogroup $\mathrm{C}_{4}$ and one to sub-genogroup $\mathrm{C}_{2}$ (Table 1 ). 
TABLE 1

Number of enterovirus surveillance samples analysed and enterovirus A71 subtyping results, Germany, 2016-July 2019

\begin{tabular}{|l|c|c|c|c|}
\hline EVSurv samples tested & $\begin{array}{c}2016 \\
(\mathrm{n})\end{array}$ & $\begin{array}{c}2017 \\
(\mathrm{n})\end{array}$ & $\begin{array}{c}2018 \\
(\mathrm{n})\end{array}$ & $\begin{array}{c}\text { Jan-Jul } 2019 \\
(\mathrm{n})\end{array}$ \\
\hline Total & 2,444 & 2,188 & 1,959 & 1,160 \\
\hline EV-positive & 527 & 448 & 416 & 209 \\
\hline EV-typed & 445 & 380 & 349 & 136 \\
\hline EV-A71-positive & 77 & 32 & 40 & 38 \\
\hline EV-A71 sub-genogroups & 57 & 18 & 23 & 21 \\
\hline Lineage C1-like & 10 & 6 & 0 & 1 \\
\hline C2 & 0 & 0 & 12 & 4 \\
\hline C4 & 10 & 8 & 5 & 12 \\
\hline ND & & & & \\
\hline
\end{tabular}

EV: enterovirus; EVSurv: enterovirus surveillance; Jan: January; Jul: July; ND: not determined.

Number of EV-A71 strains and sub-genogroup assignment based on the VP1 region is shown.

\section{Epidemiological data}

EV-A71-positive samples were reported from 11 of 16 federal states in Germany, with patients hospitalised in 20 different hospitals. All patients were between o and 10 years old, with 30 of 38 between o and 5 years old. Of these, 8 were younger than 1 year old. Twentyone were male and 17 were female. All EV-A71 strains were detected in stool samples.

\section{Phylogenetic relation}

Sequence analysis of C1-like strains identified in 2019 showed high nucleotide (nt) identities with recently detected strains, indicating a rapid widespread circulation of this new recombinant variant [4]. Phylogenetic tree calculation showed several lineages either grouping separately or clustering to recent strains detected in France, Poland, Spain, Denmark, the United States (US) and Japan (Figure 1A).

Sequence analysis of sub-genogroup $\mathrm{C}_{4}$ showed separate clustering of the German EV-A71 $\mathrm{C}_{4}$ strains detected in 2011-2013 and 2018-July 2019. Currentlycirculating strains grouped together with EV-A $71 \mathrm{C}_{4}$ strains detected in south-eastern Asia (Figure 1B) with highest nt identities to $\mathrm{MH}_{716155}, \mathrm{MH}_{716157}$ and MG431943 detected in patients with severe HFMD. This cluster also includes strains isolated from patients with severe HFMD and even fatal courses (KT428649, KT428650).

\section{Enterovirus A71 detections in routine samples}

The increased circulation of EV-A71 in 2019 has also been indicated by samples routinely sent to the NRZ PE, mostly from other laboratories sending in EV-positive samples for typing, but also from clinicians/hospitals not yet participating in EVSurv or those submitting samples from patients with diseases other than suspected aseptic meningitis/encephalitis or acute flaccid paralysis/myelitis. Of $126 \mathrm{EV}$ strains typed from January through July 2019, 23 were assigned to EV-A71. Of these, 19 were assigned to EV-A71 lineage C1-like, three to EV-A71 sub-genogroup $\mathrm{C}_{4}$ and one to EV-A71 sub-genogroup C2 (Table 2). Notably, the number of EV-A71 detections in the first 7 months of $2019(n=23)$ has already exceeded the total of such detections for 2017 and 2018, as well as the totals from the epidemic years of 2010 (6/61), 2013 (12/206) and 2016 (17/144).

Germany does not conduct standardised HFMD surveillance, but occasionally, samples collected within nursery school outbreaks of HFMD are submitted to the NRZ PE by the local health authorities. From 2010 until 2018, between 15 and 82 samples from HFMD patients were submitted per year, with 6 to 57 patients being positive for EV and 2 to 8 patients being positive for EV-A71 annually. In 2018, there were 24 HFMD patients positive for EV, eight of whom had EV-A71. Of those eight, EV-A71 $\mathrm{C}_{4}$ was detected for the first time in four children with HFMD. For two strains $(17672,17673)$, the VP1 region was sequenced and compared with EV-A71 $\mathrm{C}_{4}$ strains identified in samples analysed within the EVSurv in 2018 and 2019. Close clustering indicates that there is no relationship between the VP1 region sequence and the presence of HFMD or symptoms related to aseptic meningitis/encephalitis (Figure 1B).

\section{Discussion and conclusion}

Our findings suggest an increased circulation of EV-A71 strains in Germany already in the first 7 months of the year. Considering the fact that the EV season is still ongoing, the data available suggest that 2019 will become an epidemic year similar to 2016, 2013 and 2010.

The emergence of new EV variants exhibiting altered antigenic sites might result in increased circulation and/ or changed clinical manifestation. In Europe, EV-A71 was only sporadically associated with neurological disease during the past 30 years. In Germany, the predominant EV-A71 sub-genogroup C 2 was replaced by $C_{1}$-like strains in 2016. Emergence of EV-A71 sub-genogroup $\mathrm{C}_{4}$ strains in Germany was detected between 2011 and 2013 [2], with France [10], Russia [11] and Denmark [12] also reporting such detection around the same time.

One limitation of the EVSurv is that no detailed clinical information is available since the EVSurv request form deliberately only asks for basic cardinal symptoms: fever, nuchal rigidity, headache and vomiting. Since non-polio EV infections are not mandatorily notifiable at federal level in Germany, no data on severe cases or deaths are available. No routine testing for EV is performed in deaths so we therefore cannot exclude fatal cases with other EV than EV-A71. Nevertheless, we were informed of an additional fatal case where EV-A71 was detected in a German child hospitalised in Austria in July 2019 (personal communication, Franz Allerberger 
FIGURE 1

Phylogenetic trees of (A) sub-genogroup C1, including lineage C1-like and (B) sub-genogroup C4, 2019

A. Sub-genogroup C1, including lineage C1-like

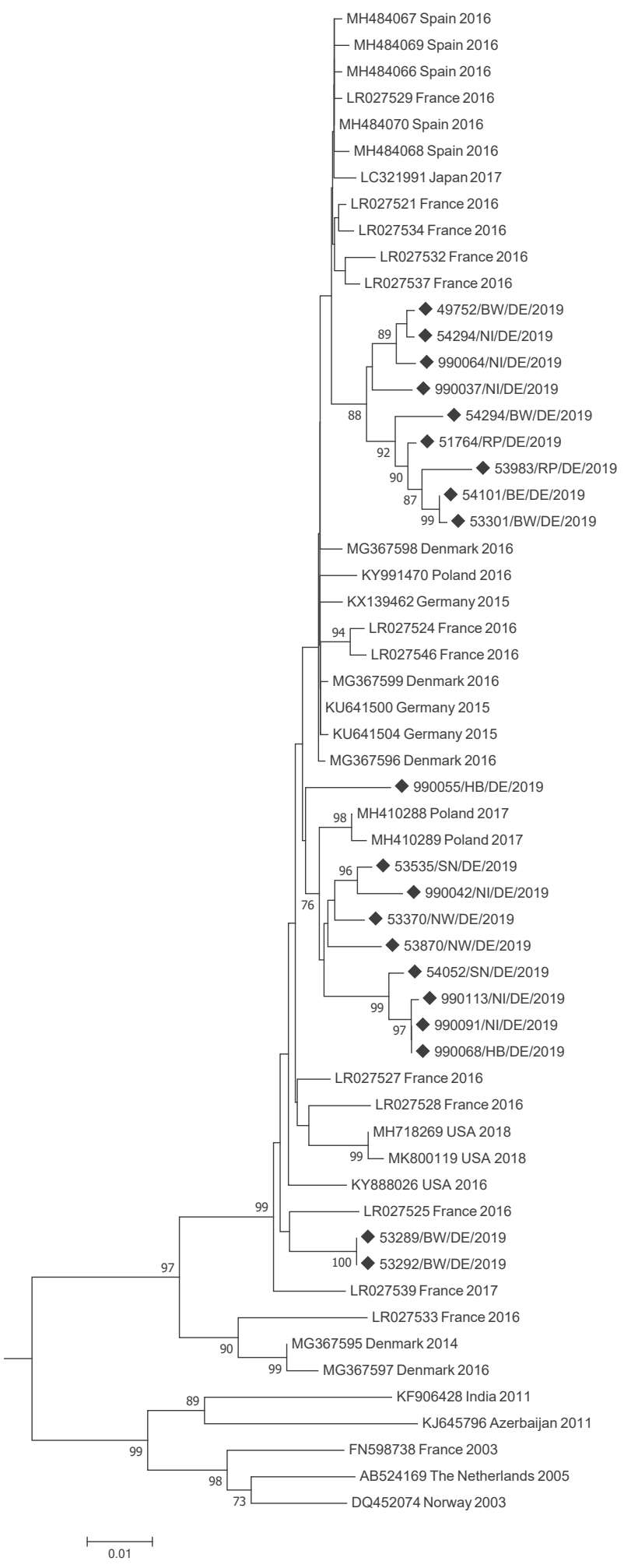

B. Sub-genogroup C4

The phylogenetic tree is based on complete viral protein 1 (VP1) nucleotide (nt) sequences (891 b corresponding to nt position $2439-3329$ in the prototype $\mathrm{BrCr}$ ETU22521). Strains identified within the German enterovirus surveillance (EVSurv) and a representative set of enterovirus A71 strains retrievable from GenBank were used. Only sequences covering the complete VP1 region were included in the analysis. Subtrees including only EV-A71 sub-genogroup $C_{1}$, including lineage C1-like (A) and EV-A71 sub-genogroup C 4 (B) are shown. Strains identified within the EVSurv are marked with filled diamonds and strains identified in hand, foot and mouth disease (HFMD) patients with open diamonds. The tree was calculated using the neighbour-joining algorithm with 1,000 replicates in MEGA software version 7.0.26. Only bootstrap values $>70$ are shown. Scale bars indicate nt substitution per site. 
Number of samples sent to the National Reference Centre for Poliomyelitis and Enteroviruses for enterovirus detection and typing, Germany, 2010-July 2019

\begin{tabular}{|c|c|c|c|c|c|c|c|c|c|c|}
\hline NRZ PE samples tested & $\begin{array}{c}2010 \\
(n)\end{array}$ & $\begin{array}{l}2011 \\
(n)\end{array}$ & $\begin{array}{c}2012 \\
(\mathrm{n})\end{array}$ & $\begin{array}{c}2013 \\
\text { (n) }\end{array}$ & $\begin{array}{c}2014 \\
(n)\end{array}$ & $\begin{array}{c}2015 \\
\text { (n) }\end{array}$ & $\begin{array}{c}2016 \\
(n)\end{array}$ & $\begin{array}{c}2017 \\
\text { (n) }\end{array}$ & $\begin{array}{c}2018 \\
\text { (n) }\end{array}$ & $\begin{array}{c}\text { Jan-Jul } 2019 \\
\text { (n) }\end{array}$ \\
\hline Total & 128 & 78 & 137 & 279 & 166 & 153 & 252 & 279 & 278 & 213 \\
\hline EV-positive & 61 & 58 & 59 & 206 & 93 & 81 & 144 & 147 & 181 & 126 \\
\hline EV-A71 positive & 6 & 1 & 7 & 12 & 2 & 12 & 17 & 6 & 7 & 23 \\
\hline \multicolumn{11}{|l|}{ EV-A71 sub-genogroup } \\
\hline $\mathrm{C}_{1}$ & 0 & 0 & 1 & 3 & 1 & 0 & 0 & 0 & 0 & 0 \\
\hline Lineage $C_{1}$-like & 0 & 0 & 0 & 0 & 0 & 7 & 9 & 2 & 7 & 19 \\
\hline $\mathrm{C}_{2}$ & 6 & 1 & 1 & 9 & 0 & 4 & 8 & 4 & 0 & 1 \\
\hline $\mathrm{C}_{4}$ & 0 & 0 & 5 & 0 & 1 & 1 & 0 & 0 & 0 & 3 \\
\hline
\end{tabular}

EV: enterovirus; Jan: January; Jul: July; NRZ-PE: National Reference Centre for Poliomyelitis and Enteroviruses.

Number of EV-A71 strains and sub-genogroup assignment based on the VP1 region is shown.

and Birgit Prochazka, August 2019). Taken together with the fatal case reported in the EVSurv, this raises concern about increased occurrence of severe cases as a consequence of increased EV-A71 circulation.

Reports of severe clinical presentation associated with detection of a EV-A71 C1-like strains [3,5-7], together with recent indications of long-term sequelae $[13,14]$, should be a signal for physicians and public health authorities to include/intensify EV diagnostic in patients showing signs of aseptic meningitis, encephalitis or acute flaccid paralysis/myelitis.

Continued virological data could provide first hints to upcoming public health matters. (EV typing should be conducted to enable molecular epidemiology. Furthermore, systematic studies are needed to assess the burden of EV infections in Europe.

Laboratory Network for Enterovirus Diagnostic (LaNED) Nikolaus Ackermann, Maja Adam, Armin Baillot, Ingrid Ehrhard, Martin Enders, Wali Hafezi, Ralph Hahn, Jörg Hofmann, Klaus Korn, Joachim Kühn, Detlef Michel, Alexandra Pettke, Christiane Prifert, Holger F. Rabenau, Ursula Reif, Gabriele Rieder, Benedikt Weißbrich, Andreas Wille and Marta J Zuchowski.

\section{Acknowledgements}

We thank all clinicians participating in the German enterovirus surveillance; all cooperating labs sending enterovirus strains for typing; the local health authorities for collecting samples from hand, foot and mouth disease patients; and the technical staff in all labs for excellent performance.

Funding statement: Results were obtained during routine work under the auspices of the German Federal Ministry of Health.

\section{Conflict of interest}

None declared.

\section{Authors' contributions}

Members of the Laboratory Network (LaNED) analysed samples at the laboratory level. KK coordinated the EVSurv and collected the data. KK, SD and SB analysed the data. SB wrote the manuscript draft, all co-authors commented and approved the final version.

\section{References}

1. Hassel C, Mirand A, Lukashev A, TerletskaiaLadwig E, Farkas A, Schuffenecker I, et al. Transmission patterns of human enterovirus 71 to, from and among European countries, 2003 to 2013. Euro Surveill. 2015;20(34):30005. https://doi. org/10.2807/1560-7917.ES.2015.20.34.30005 PMID: 26530407

2. Böttcher S, Obermeier PE, Neubauer K, Diedrich SLaboratory Network for Enterovirus Diagnostics. Recombinant Enterovirus A71 Subgenogroup C1 Strains, Germany, 2015. Emerg Infect Dis. 2016;22(10):1843-6. https://doi.org/10.3201/ eid2210.160357 PMID: 27439117

3. Karrasch M, Fischer E, Scholten M, Sauerbrei A, Henke A, Renz DM, et al. A severe pediatric infection with a novel enterovirus A71 strain, Thuringia, Germany. I Clin Virol. 2016;84:90-5. https://doi.org/10.1016/j.jcv.2016.09.007 PMID: 27771495

4. Ngangas ST, Lukashev A, Jugie G, Ivanova O, Mansuy JM, Mengelle C, et al. Multirecombinant Enterovirus A71 Subgenogroup $C_{1}$ Isolates Associated with Neurologic Disease, France, 2016-2017. Emerg Infect Dis. 2019;25(6):1204-8. https://doi.org/10.3201/eid2506.181460 PMID: 31107209

5. Wieczorek M, Purzyńska M, Krzysztoszek A, Ciąćka A, Figas A, Szenborn L. Genetic characterization of enterovirus A71 isolates from severe neurological cases in Poland. J Med Virol. 2018;90(2):372-6. https://doi.org/10.1002/jmv.24958 PMID: 28960454

6. Antona D, Kossorotoff M, Schuffenecker I, Mirand A, LeruezVille M, Bassi C, et al. Severe paediatric conditions linked with EV-A71 and EV-D68, France, May to October 2016. Euro Surveill. 2016:21(46):30402. https://doi.org/10.2807/15607917.ES.2016.21.46.30402 PMID: 27918268

7. Casas-Alba D, de Sevilla MF, Valero-Rello A, Fortuny C, GarcíaGarcía JJ, Ortez C, et al. Outbreak of brainstem encephalitis associated with enterovirus-A71 in Catalonia, Spain (2016): a clinical observational study in a children's reference centre in Catalonia. Clin Microbiol Infect. 2017;23(11):874-81. https:// doi.org/10.1016/j.cmi.2017.03.016 PMID: 28344164

8. González-Sanz R, Casas-Alba D, Launes C, Muñoz-Almagro C, Ruiz-García MM, Alonso M, et al. Molecular epidemiology of an enterovirus A71 outbreak associated with severe neurological disease, Spain, 2016. Euro Surveill. 2019;24(7):1800089. https://doi.org/10.2807/1560-7917.ES.2019.24.7.1800089 PMID: 30782267

9. Kroneman A, Vennema H, Deforche K, v d Avoort H, Peñaranda $\mathrm{S}$, Oberste MS, et al. An automated genotyping tool for enteroviruses and noroviruses. J Clin Virol. 2011;51(2):121-5. https://doi.org/10.1016/j.jcv.2011.03.006 PMID: 21514213

10. Schuffenecker I, Henquell C, Mirand A, Coste-Burel M, MarqueJuillet S, Desbois D, et al. New introductions of enterovirus 
71 subgenogroup $C_{4}$ strains, France, 2012. Emerg Infect Dis. 2014;20(8):1343-6. https://doi.org/10.3201/eid2008.131858 PMID: 25061698

11. Akhmadishina LV, Govorukhina MV, Kovalev EV,

Nenadskaya SA, Ivanova OE, Lukashev AN. Enterovirus A71

Meningoencephalitis Outbreak, Rostov-on-Don, Russia, 2013 Emerg Infect Dis. 2015;21(8):1440-3. https://doi.org/10.3201/ eid2108.141084 PMID: 26196217

12. Fischer TK, Nielsen AY, Sydenham TV, Andersen PH, Andersen $B$, Midgley SE. Emergence of enterovirus 71 C4a in Denmark, 2009 to 2013. Euro Surveill, 2014:19(38):20911. https://doi. org/10.2807/1560-7917.ES2014.19.38.20911 PMID: 25306878

13. Huang MC, Wang SM, Hsu YW, Lin HC, Chi CY, Liu CC.

Long-term cognitive and motor deficits after enterovirus

71 brainstem encephalitis in children. Pediatrics.

2006;118(6):e1785-8. https://doi.org/10.1542/peds.2006-1547 PMID: 17116698

14. Chang LY, Lin HY, Gau SS, Lu CY, Hsia SH, Huang YC, et al. Enterovirus A71 neurologic complications and long-term sequelae. J Biomed Sci. 2019;26(1):57. https://doi.org/10.1186/ S12929-019-0552-7 PMID: 31395054

\section{License, supplementary material and copyright}

This is an open-access article distributed under the terms of the Creative Commons Attribution (CC BY 4.0) Licence. You may share and adapt the material, but must give appropriate credit to the source, provide a link to the licence and indicate if changes were made.

Any supplementary material referenced in the article can be found in the online version.

This article is copyright of the authors or their affiliated institutions, 2019. 